Curtius: Die besonderen Reaktionen etc. 323

Körper noch drei 4-Methyl.5-äthylpyrazolinkerne; wie aus obigem hervorgeht, zeigt die Verbindung in der Tat alle Realtionen des einfachen 4-Methyl-5-äthylpyrazolins.

Säuert man nach Abscheidung der beiden Pyrazoline die alkalische Flüssigkeit mit verdünnter Schwefelsäure an, so fällt Fumarsäure ${ }^{1)}$ aus. Der daraus zur Charakterisierung dargestellte $\mathrm{Fumarsäuredimethylester}{ }^{2}$ ) krystallisierte in den bekannten langen Prismen vom Schmp. $103^{\circ}$.

$0,1261 \mathrm{~g}$ gaben $0,2299 \mathrm{~g} \mathrm{CO}_{2}$ und $0,0641 \mathrm{~g} \mathrm{H}_{2} \mathrm{O}$.

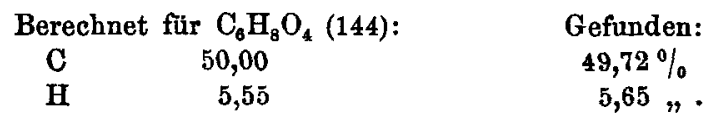

Der Dimethylester wurde endlich noch mit starkem, wäbrigem Ammoniak längere Zeit stehen gelassen, wobei Fumaramid ${ }^{3}$ ) als sandiges Pulver erhalten wurde.

\title{
III. Hydrazid und AzId der Benzylmalonsüure.
}

[Nach Versuchen von 0wen E. Mott. $\left.\left.{ }^{4}\right)\right]$

$$
\begin{aligned}
& \text { Benzyl-malonsäure-dihydrazid, } \\
& \mathrm{C}_{6} \mathrm{H}_{6} . \mathrm{CH}_{2} . \mathrm{CH}\left\langle\begin{array}{l}
\mathrm{CO} . \mathrm{NH} . \mathrm{NH}_{2} \\
\mathrm{CO} . \mathrm{NH} . \mathrm{NH}_{2}
\end{array} .\right.
\end{aligned}
$$

$100 \mathrm{~g}$ Benzylmalonsäurediäthylester, der am besten nach dem schönen, neuen Verfahren von Leuchs ${ }^{5}$ ) dargestellt wird,

इ) Vgl. Foersterling, dies. Journ. [2] 51, 395 (1895).

2) Ebenda.

9) Curtius u. Koch, dies. Journ. [2] 38, 478 (1888).

4) Owen E. Mott, „Über Benzylmalonhydrazid “. Inaug.-Diss. Heidelberg 1900. Druck von E. Geisendörfer. Die Arbeit enthält, wie mir der Verfasser mehrere Jahre nach seiner Promotion freiwillig eingestanden, zahlreiche falsche Analysen. Ich habe darauf die ganze Untersuchung in meinem Institut durch Herrn Wilhelm Sieber unter Leitung von Herrn Prof. Darapsky wiederholen lassen. Es gelang dabeí, die Ausbeute an Azid aus dem Hydrazid beträchtlich zu steigern sowie ferner das Urethan fast quantitativ in Pbenylacetaldehyd überzuführen. Ich gebe darum im folgenden nur die neuen Beobachtungen von Sieber wieder, ohne weiter auf die leider fast durchweg unzuverlässigen Angaben von Mott einzugehen. Curtius.

3) Ber. 44, 1509 (1911). 


\section{Curtius: Die besonderen Reaktionen etc.}

werden mit $50 \mathrm{~g}$ Hydrazinhydrat unter Zusatz ron $10 \mathrm{ccm}$ absolutem Alkohol 6 Stunden lang im Schliffkolben unter Rückflub auf dem Wasserbad erhitzt. Nach dem Erkalten wird die erhaltene weiße Krystallmasse abgesaugt, mit wenig Alkohol und nachher mit Ather gewaschen und auf Ton im Vakuum getrocknet. Die Ausbeute an Rohprodukt beträgt 91,5 g. Durch Umkrystallisieren aus heißem Wasser erhält man reines Benzylmalonyldihydrazid in feinen, glänzenden Nadeln, die bei $164^{\circ}$ schmelzen. Die Substanz ist in Wasser leicht, in Alkohol schwer löslich und in Äther unlöslich. Aus der warmen alkoholischen Lösung scheidet sich das Hydrazid beim Erkalten zunächst als gallertartige Masse aus, die sich erst nach einiger Zeit infolge Bildung von Krystallen trübt.

$0,1099 \mathrm{~g}$ gaben $0,2194 \mathrm{~g} \mathrm{CO}$ und $0,0673 \mathrm{~g} \mathrm{H}_{2} \mathrm{O}$.

$0,0448 \mathrm{~g}$ gaben $10,0 \mathrm{~cm} \mathrm{~N}$ bei $18^{\circ}$ und $750 \mathrm{~mm}$.

$\begin{array}{ccc}\text { Berechnet für } \mathrm{C}_{10} \mathrm{H}_{14} \mathrm{O}_{2} \mathrm{~N}_{4}(222): & \text { Gefunden: } \\ \mathrm{C} & 54,05 & 54,45 \% \\ \mathrm{H} & 6,31 & 6,80 \% \\ \mathrm{~N} & 25,22 & 25,44 \%\end{array}$

Dibydrochlorid. $30 \mathrm{~g}$ obigen Hydrazids werden in $150 \mathrm{ccm}$ Wasser gelöst und unter Eiskühlung zunächst 200 bis $250 \mathrm{ccm}$ alkoholische Salzsäure und sodann das gleiche Volumen Äther hinzugefügt. Der ausgeschiedene Krystallbrei wird nach 1-2 stündigem Stehen abgesaugt, mit Äther gewaschen und im Vakuum getrocknet. Ausbeute bis zu $93 \%$ der Theorie. Das Rohprodukt schmilzt, frisch dargestellt, bei $135^{\circ}$; beim Aufbewahren steigt der Schmelzpunkt, indem das Salz spontan unter Abspaltung ron Diammoniumchlorid in cyclisches sekundäres Benzylmalonylhydrazid (vgl. S. 325) übergeht. Das Salz ist spielend löslich in Wasser, leicht in Alkohol und unlöslich in Äther.

$0,1299 \mathrm{~g}$ gaben $21,7 \mathrm{~cm} \mathrm{~N}$ bei $18^{\circ}$ und $750 \mathrm{~mm}$.

$0,1068 \mathrm{~g}$ gaben $0,1052 \mathrm{~g} \mathrm{AgCl}$.

Berechnet für $\mathrm{C}_{10} \mathrm{H}_{14} \mathrm{O}_{2} \mathrm{~N}_{4}, 2 \mathrm{HCl}$ (295): Gefunden:

$\begin{array}{lll}\mathrm{N} & 18,98 & 19,04 \% \\ \mathrm{Cl} & 24,07 & 24,37 \%\end{array}$

Dibenzalverbindung. Fällt beim Schütteln des Dihydrazids in wäBriger Lösung mit dem gleichen Gewicht Benzaldehyd als weiBe, flockige Masse aus und bildet nach zwei- 
Curtius: Die besonderen Reaktionen etc. 325

maligem Umkrystallisieren aus 50 prozent. Essigsäure kleine Nadeln vom Schmp. 220 . Das Produkt ist in Eisessig leicht, in Alkohol schwer, in Benzol noch weniger löslich und in Äther ganz unlöslich.

$0,1421 \mathrm{~g}$ gaben $0,3773 \mathrm{~g} \mathrm{CO}$, und $0,0707 \mathrm{~g} \mathrm{H}_{\mathbf{2}} \mathrm{O}$.

$0,0901 \mathrm{~g}$ gaben $11,3 \mathrm{ccm} N$ bei $19^{\circ}$ und $748 \mathrm{~mm}$.

$\begin{array}{ccc}\text { Berechnet für } \mathrm{C}_{24} \mathrm{H}_{22} \mathrm{O}_{2} \mathrm{~N}_{4}(398): & \text { Gefunden: } \\ \mathbf{C} & \mathbf{7 2 , 3 6} & \mathbf{7 2 , 4 2} \% \\ \mathbf{H} & 5,53 & 5,53 \% \\ \mathrm{~N} & 14,07 & 14,18 \%\end{array}$

Sekundäres Benzyl-malonsäure-hydrazid, $\mathrm{C}_{6} \mathrm{H}_{5} \cdot \mathrm{CH}_{2} \cdot \mathrm{CH}\left\langle\begin{array}{l}\mathrm{CO} . \mathrm{NH} \\ \mathrm{CO} . \mathrm{NH}\end{array}\right.$.

1. Aus salzsaurem Benzyl-malonsäure-dihydrazid durch Erhitzen.

$10 \mathrm{~g}$ trockenes salzsaures Hydrazid werden, auf einem Uhrglas ausgebreitet, 4-5 Stunden lang auf dem Wasserbad erhitzt. Der Rückstand wird mit wenig kaltem Wasser angerührt, abgesaugt und zweimal aus heiBem 50 prozent. Alkohol umkrystallisiert. Man erhält so das sekundäre Hydrazid in farblosen, glänzenden Blättchen vom Schmp. 2410. Die Ausbeute beträgt $5,9 \mathrm{~g}$, entsprechend $92 \%$ der Theorie. Die Substanz ist in Alkohol löslich, sehr leicht in heißem und sehr schwer in kaltem Wasser, unlöslich in Äther oder Ligroin.

Die gleiche Verbindung wird auch bei mehrtägigem Aufbewahren des salzsauren Hydrazids besonders in feuchtem $\mathrm{Zu}$ stande erhalten. Diese Entstehung entspricht ganz der von Jordan ${ }^{1}$ ) beobachteten Bildung von sekundärem Phenylpropionsäurehydrazid bei längerem Stehen der wäBrigen Lösung des salzsauren primären Hydrazids.

2. Aus Benzyl-malonsäure-dihydrazid und Jod.

$5 \mathrm{~g}$ Hydrazid werden in $30 \mathrm{~g}$ Alkohol gelöst und auf dem Wasserbad allmählich mit einer konzentrierten alkoholischen Lösung von 2,7g Jod versetzt. Bei jedesmaligem Zusatz einer

1) Dies. Journ. [2] 64, 305 (1901). 
326 Curtius: Die besonderen Reaktionen etc.

neuen Jodmenge tritt Gasentwicklung auf. Nachdem die Hälfte des Jods eingetragen, beginnt die Abscheidung des sekundären Hydrazids. Man erhitzt die Mischung noch eine halbe Stunde lang auf dem Wasserbade weiter, saugt dann $a b$ und wäscht mit Wasser gut aus.

Statt in alkoholischer Lösung zu arbeiten, kann man das primäre Hydrazid auch in wenig Wasser auflösen und Jodtinktur wie vorher hinzufügen. Dampft man nach beendigter Reaktion die Hauptmenge des Wassers und Alkohols ab, so fällt das sekundäre Hydrazid aus.

\section{Aus Benzyl-malonsäure-dihydrazid und} salpetriger Säure.

Das sekundäre Hydrazid entsteht endlich auch als Nebenprodukt bei der Darstellung des Azids (vgl. S. 276), aus dem primären Hydrazid und salpetriger Säure, besonders beim Arbeiten in schwach mineralsaurer Lösung (vgl. S. 327).

Die auf allen drei Wegen erhaltenen Produkte waren miteinander identisch. Sie zeigten nach dem Umkrystallisieren aus 50 prozent. Alkohol den gleichen Schmp. $241^{\circ}$ und auch bei der Analyse die gleiche Zusammensetzung:

I. Substanz nach 1. dargestellt.

$0,1552 \mathrm{~g}$ gaben $0,3591 \mathrm{~g} \mathrm{CO}_{2}$ und $0,0743 \mathrm{~g} \mathrm{H}_{2} \mathrm{O}$.

$0,1155 \mathrm{~g}$ gaben $15,6 \mathrm{ccm} \mathrm{N}$ bei $25^{\circ}$ und $756 \mathrm{~mm}$.

II. Substanz nach 2. dargestellt.

$0,1225 \mathrm{~g}$ gaben $0,2829 \mathrm{~g} \mathrm{CO}_{2}$ und $0,0595 \mathrm{~g} \mathrm{H}_{2} \mathrm{O}$.

$0,1152 \mathrm{~g}$ gaben $15,1 \mathrm{ccm} \mathrm{N}$ bei $15^{\circ}$ und $742 \mathrm{~mm}$.

III. Substanz nach 3. dargestellt.

$0,2010 \mathrm{~g}$ gaben $0,4679 \mathrm{~g} \mathrm{CO}_{2}$ und $0,0967 \mathrm{~g} \mathrm{H}_{2} \mathrm{O}$.

$0,1198 \mathrm{~g}$ gaben $15,87 \mathrm{~cm} \mathrm{~N}$ bei $20^{\circ}$ und $756 \mathrm{~mm}$.

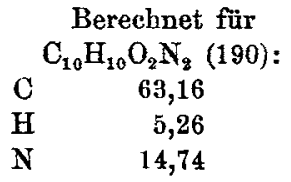

\begin{tabular}{rrr}
\multicolumn{4}{c}{ Gefunden: } \\
I. & II. & III. \\
63,10 & 62,98 & $63,49 \%$ \\
5,32 & 5,40 & $5,26 \%$ \\
14,98 & 14,98 & $15,00 \%$.
\end{tabular}

Das Molekulargewicht wurde durch Erniedrigung des Gefrierpunktes einer Lösung der Substanz in Eisessig bestimmt. 
Curtius: Die besonderen Reaktionen etc. 327

I. Substanz nach 1. dargestellt.

$0,1210 \mathrm{~g}$ gaben in $13,63 \mathrm{~g}$ Eisessig $0,198^{\circ}$ Depression.

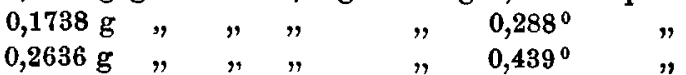

II. Substanz nach 2. dargestellt.

$0,1319 \mathrm{~g}$ gaben in $12,51 \mathrm{~g}$ Eisessig $0,237^{\circ}$ Depression.

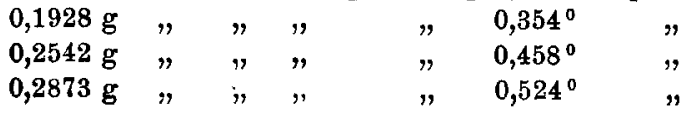

\begin{tabular}{ccrrrrrrr}
\multicolumn{3}{c}{ Berechnet für } & \multicolumn{5}{c}{ Gefunden: } \\
& $\mathrm{C}_{10} \mathrm{H}_{10} \mathrm{O}_{3} \mathrm{~N}_{2}:$ & & $\mathrm{I}$. & & \multicolumn{3}{c}{ II. } \\
M & 190 & 175 & 173 & $172 ;$ & 174 & 170 & 173 & 171.
\end{tabular}

Benzyl-malonsäure-diazid,<smiles></smiles>

Salzsaures Benzylmalonsäuredihydrazid (1 Mol.) wird in wenig Wasser gelöst, mit Äther überschichtet und unter Rühren und Eiskühlung eine konzentrierte Lösung von Natriumnitrit (2 Mol.) langsam zuflieBen gelassen. Das entstehende Azid wird dabei vom Äther aufgenommen, während das daneben gebildete sekundäre Hydrazid (vgl. S. 326) in weißen Blättchen aus der wäBrigen Lösung ausfällt. Nachdem alles Nitrit eingetragen, wird der gelb gefärbte Äther abgegossen, die wäBrige Flüssigkeit noch einmal mit Äther durchgeschüttelt, die vereinigten ätherischen Auszüge mit wenig Wasser gewaschen und dann bei $0^{0}$ eine Stunde lang über entwässertem Natriumsulfat getrocknet. Beim Abblasen des Äthers hinterbleibt das Azid als gelbes Öl; das in Alkohol und Äther löslich, in Wasser unlöslich ist und beim Erhitzen lebhaft verpufft. Das in der wäBrigen Lösung abgeschiedene sekundäre Hydrazid wird abgesaugt, mit Wasser gewaschen und getrocknet. Aus $35 \mathrm{~g}$ salzsaurem Salz wurden so $5 \mathbf{g}$ sekundäres Hydrazid erhalten. Die Bildung dieses Nebenproduktes wird bei Gegenwart überschüssiger Mineralsäure entsprechend folgender Vorschrift beinahe völlig verhindert:

$11,1 \mathrm{~g}$ Benzylmalonsäuredihydrazid werden in einer wieder erkalteten Mischung von 9,8 $\mathrm{g}$ konzentrierter Schwefelsäure und $44 \mathrm{ccm}$ Wasser gelöst, mit $50 \mathrm{ccm}$ Äther versetzt und bei $-5^{\circ}$ 
328 Curtius: Die besonderen Reaktionen etc.

unter gleichzeitigem Durchruhren der beiden Schichten eine Lösung von 10,35 g Natriumnitrit in $21 \mathrm{ccm}$ Wasser tropfenweise hinzugegeben. Die weitere Behandlung ist die gleiche wie oben.

$$
\begin{aligned}
& \text { Benzyl-malonsäure-dianilid, } \\
& \mathrm{C}_{6} \mathrm{H}_{6} \cdot \mathrm{CH}_{2} \cdot \mathrm{CH} \backslash \begin{array}{l}
\mathrm{CO} . \mathrm{NH} . \mathrm{C}_{6} \mathrm{H}_{5} \\
\mathrm{CO} . \mathrm{NH} . \mathrm{C}_{6} \mathrm{H}_{6}
\end{array}
\end{aligned}
$$

Die äthèrische Lösung des Azids aus $5 \mathrm{~g}$ salzsaurem Hydrazid wird mit $6,3 \mathrm{~g}$ frisch destilliertem Anilin versetzt und auf dem Wasserbad am Rückflußkühler erhitzt. Gleich beim Anwärmen ist Geruch nach Stickstoffwasserstoff wahrzunehmen. Nach 4 stündigem Kochen wird der Äther verdampft und das zurückbleibende Anilid aus Alkohol umkrystallisiert. Schmelzpunkt $217^{\circ}$.

$0,1482 \mathrm{~g}$ gaben $0,4162 \mathrm{~g} \mathrm{CO}_{2}$ und $0,0800 \mathrm{~g} \mathrm{H}_{2} \mathrm{O}$.

$0,1296 \mathrm{~g}$ gaben $9,3 \mathrm{ccm} \mathrm{N}$ bei $13^{\circ}$ und $740 \mathrm{~mm}$.

$\begin{array}{ccc}\text { Berechnet für } \mathrm{C}_{22} \mathrm{H}_{20} \mathrm{O}_{2} \mathrm{~N}_{2} \text { (344): } & \text { Gefunden: } \\ \mathrm{C} & 76,74 & 76,59 \% \\ \mathrm{H} & 5,81 & 6,00 \% \\ \mathrm{~N} & 8,14 & 8,25 \%\end{array}$

Benzyl-methylen-diurethan,

$$
\mathrm{C}_{6} \mathrm{H}_{5} \cdot \mathrm{CH}_{2} \cdot \mathrm{CH}\left\langle\begin{array}{l}
\mathrm{NH} . \mathrm{CO}_{2} \mathrm{C}_{2} \mathrm{H}_{5} \\
\mathrm{NH} \cdot \mathrm{CO}_{2} \mathrm{C}_{2} \mathrm{H}_{6}
\end{array}\right. \text {. }
$$

Die über Natriumsulfat getrocknete ätherische Lösung des Azids aus $11,1 \mathrm{~g}$ Benzylmalonyldihydrazid (vgl. S. 327) wird mit dem doppelten Volumen absoluten Alkohols 3 Stunden lang auf dem Wasserbad erhitzt. Nach beendeter Stickstoffentwicklung werden der 太̈ther und die Hauptmenge des Alkohols abdestilliert. Der Rückstand erstarrt beim Stehen im Vakuumexsiccator zu einer gelblich weiben, krystallinischen Masse. Ausbeute: 10,5 g. Durch Umkrystallisieren aus wenig heißem Alkohol erhält man reines Benzylmethylendiurethan in äuBerst feinen, weißen, filzigen Nadeln vom Schmp. $166^{\circ}$. Es ist in Ather und Alkohol leicht, in Benzol schwerer löslich, und in Wasser und Ligroin unlöslich.

$0,1326 \mathrm{~g}$ gaben $0,2944 \mathrm{~g} \mathrm{CO}$ und $0,0868 \mathrm{~g} \mathrm{H}_{2} \mathrm{O}$.

$0,0988 \mathrm{~g}$ gaben $9,3 \mathrm{ccm} \mathrm{N}$ bei $25^{\circ}$ und $742 \mathrm{~mm}$. 
Curtius: Die besonderen Reaktionen etc. 329

$\begin{array}{ccc}\text { Berechnet für } \mathrm{C}_{14} \mathrm{H}_{20} \mathrm{O}_{4} \mathrm{~N}_{2}(280): & \text { Gefunden: } \\ \text { C } & 60,00 & 60,55 \% \\ \mathrm{H} & 7,14 & 7,27 \% \\ \mathrm{~N} & 10,00 & 10,24 \%\end{array}$

Phenylacetaldehyd, $\mathrm{C}_{6} \mathrm{H}_{6} \cdot \mathrm{CH}_{2} \cdot \mathrm{CHO}$; aus Benzyl-methylen-diurethan.

Das Urethan wurde zuerst mit überschüssiger 25 prozent. Schwefelsäure zersetzt; die Ausbeute an Aldehyd ist aber unter diesen Bedingungen nur sehr gering, indem der Aldehyd durch die Mineralsäure offenbar gröBtenteils zu dem ron Stobbe und Lippold ${ }^{1}$ ) in neuerer Zeit untersuchten Triphenylparaldehyd polymerisiert wird. Wendet man dagegen nur die berechnete Menge 2 prozent. Schwefelsäure an, so wird die Ausbeute nahezu quantitativ:

$4,2 \mathrm{~g}$ Benzylmethylendiurethan werden mit $50 \mathrm{~g} 2$ prozent. Schwefelsäure versetzt und das Gemisch mit Wasserdampf destilliert. Der entstehende Aldehyd geht dabei als farbloses Öl von eigentümlichem Geruch mit den Wasserdämpfen über. Das Destillat wird in einer Lösung von 2,04 $\mathrm{g}$ Benzhydrazid in $100 \mathrm{ccm}$ Wasser aufgefangen, wobei sich sofort Phenylacetaldehydbenzhydrazon als weißer Niederschlag abscheidet. Ausbeute: 3,5 g Benzhydrazon, entsprechend $98 \%$ der Theorie.

Bei einem zweiten Versuch wurde das Destillat für sich aufgefangen, der Aldehyd mit Äther aufgenommen und die ätherische Lösung über entwässertem Natriumsulfat getrocknet. Nach dem Abdestillieren des Äthers wurde der ölige Rückstand im Vakuum fraktioniert. Dabei ging reiner Phenylacetaldehyd bei $81-82^{\circ}$ unter $12 \mathrm{~mm}$ Druck über. Erhalten wurden $1 \mathrm{~g}$ oder $55,5 \%$.

Zum Vergleich wurde der Aldehyd auch nach dem von $\mathrm{Rassow}$ und $\mathrm{Burmeister}{ }^{2}$ verbesserten Verfahren von Erlenmeyer sen. und $\mathrm{Lip}^{3}$ ) aus $\beta$-Phenyl- $\alpha$-chlormilchsäure dargestellt:

Die zur Umwandlung der Zimtsäure in Phenylchlormilchsäure erforderliche Lösung von Kaliumbypochlorit wurde statt

1) Dies. Journ. [2] 90, 277 (1914).

2) Dies. Journ. [2] 84, 473 (1911).

3) Ann. Chem. 219, 179 (1883). 
330 Curtius: Die besonderen Reaktionen etc.

aus Chlorkalk einfacher direkt nach der bequemen Vorschrift von $\mathrm{Graebe}^{1}$ ) bereitet. $\mathbf{7 4} \mathrm{g}$ Zimtsäure wurden mit $35 \mathrm{~g}$ Pottasche in $250 \mathrm{ccm}$ Wasser unter Erwärmen gelöst und mit einer Lösung von Kaliumhypochlorit vermischt, die durch Einleiten von Chlor aus $31,6 \mathrm{~g}$ Kaliumpermanganat und $200 \mathrm{ccm}$ konzentrierter Salzsäure in eine Lösung von $62 \mathrm{~g}$ Kaliumbydroxyd in 1 Liter Wasser hergestellt war. Die weitere Verarbeitung sowie die Überführung der Phenylchlormilchsäure in Phenylacetaldehyd erfolgte genau nach den Angaben von Rassow und Burmeister. Der so erhaltene Aldehyd zeigte den Siedepunkt $78^{\circ}$ bei $10 \mathrm{~mm}$ und war völlig identisch mit dem aus Benzylmethylendiurethan gewonnenen Produkt.

$$
\begin{gathered}
\text { Phenylacetaldehyd-benzhydrazon, } \\
\mathrm{C}_{6} \mathrm{H}_{5} . \mathrm{CH}_{2} \text {. CH:N.NH.CO. } \mathrm{C}_{6} \mathrm{H}_{5} .
\end{gathered}
$$

Bei dem Versuche, das Azin des Phenylacetaldehyds durch Schütteln einer wäBrigen Lösung von Hydrazinsulfat mit dem Aldehyd darzustellen, erhält man zunächst einen weißen, flockigen Niederschlag, der aber sofort verschmiert und sich nicht umkrystallisieren läBt. Gsnz beständig dagegen sind die Kondensationsprodukte des Aldehyds mit Benzhydrazid und mit m-Nitrobenzhydrazid.

Die Darstellung des Benzhydrazons ans dem bei der Zersetzung des Urethans mit verdünnter Schwefelsäure erhaltenen aldehydhaltigen Destillat wurde bereits oben beschrieben. Die gleiche Substanz entsteht auch aus dem reinen Aldehyd, indem man gleiche Mengen Aldehyd und Benzhydrazid in möglichst wenig absolutem Alkohol löst und das Gemisch eine halbe Stunde lang stehen läBt. Auf Zusatz von Wasser fällt das Kondensationsprodukt als weißer, krystallinischer Niederschlag aus. Durch zweimaliges Umkrystallisieren aus einer Mischung von 1 Raumteil Alkohol und 9 Raumteilen Äther erhält man farblose Nadeln, die bei $148-149^{\circ}$ schmelzen. Das Benzhydrazon ist in Alkohol sehr leicht, in Äther schwer löslich und in Wasser unlöslich.

$0,2218 \mathrm{~g}$ gaben $0,6169 \mathrm{~g} \mathrm{CO}_{2}$ und $0,1227 \mathrm{~g} \mathrm{H}_{2} \mathrm{O}$.

$0,2314 \mathrm{~g}$ gaben $24,5 \mathrm{ccm} \mathrm{N}$ bei $21^{\circ}$ und $750 \mathrm{~mm}$.

1) Ber 35, 2753 (1902). 
Curtius: Die besonderen Reaktionen ete. 331

$\begin{array}{ccc}\text { Berechnet für } \mathrm{C}_{25} \mathrm{H}_{14} \mathrm{ON}_{2} \text { (238): } & \text { Gefunden: } \\ \mathrm{C} & \mathbf{7 5 , 6 3} & \mathbf{7 5 , 8 6} \% \\ \mathrm{H} & \mathbf{5 , 8 8} & 5,91 \% \\ \mathrm{~N} & \mathbf{1 1 , 7 7} & \mathbf{1 1 , 8 2} \% \text {. }\end{array}$

Phenylacetaldehyd-m-nitrobenzhydrazon, $\mathrm{C}_{6} \mathrm{H}_{5} \cdot \mathrm{CH}_{2} \cdot \mathrm{CH}: \mathrm{N} . \mathrm{NH} . \mathrm{CO} \cdot \mathrm{C}_{6} \mathrm{H}_{4} \cdot \mathrm{NO}_{2}$.

Scheidet sich beim Zusammenbringen von $2 \mathrm{~g}$ Phenylacetaldehyd und $2,8 \mathrm{~g}$ m-Nitrobenzhydrazid in konzentrierter absolut alkoholischer Lösung nach kurzem Stehen als weißer, krystallinischer Niederschlag aus und bildet nach zweimaligem Umlösen aus heißem gewöhnlichem Alkohol kleine Krystalle vom Schmp. $152^{\circ}$.

$0,2496 \mathrm{~g}$ gaben $0,5828 \mathrm{~g} \mathrm{CO}_{2}$ und $0,1035 \mathrm{~g} \mathrm{H}_{2} \mathrm{O}$.

$0,2376 \mathrm{~g}$ gaben $30,8 \mathrm{ccm} \mathrm{N}$ bei $18^{\circ}$ und $756 \mathrm{~mm}$.

$\begin{array}{ccc}\text { Berechnet für } \mathrm{C}_{15} \mathrm{H}_{13} \mathrm{O}_{3} \mathrm{~N}_{3} \text { (283): } & \text { Gefunden: } \\ \mathrm{C} & 63,60 & 63,64 \% \\ \mathrm{H} & 4,59 & 4,64 \% \\ \mathrm{~N} & 14,84 & 14,82 \%\end{array}$

IV. Hydrazid und Azid der m-Xylylmalonsäure.

[Bearbeitet von Carlo Marangolo. $\left.\left.{ }^{\mathbf{1}}\right)\right]$

$$
\begin{gathered}
\text { m-Xylyl-malonsäuré-dihydrazid, } \\
\mathrm{CH}_{3} \cdot \mathrm{C}_{6} \mathrm{H}_{4} \cdot \mathrm{CH}_{2} \cdot \mathrm{CH} \bigcup_{\mathrm{CO} . \mathrm{NH} . \mathrm{NH}_{2}}^{\mathrm{CO}}{ }_{\mathrm{NH} . \mathrm{NH}_{2}} .
\end{gathered}
$$

Der erforderliche m-Xylylmalonsäurediäthylester

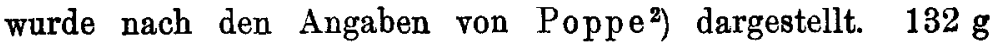
m-Xylylbromid gaben durchschnittlich $88 \mathrm{~g}$ Ester vom Siedepunkt $178-180^{\circ}$ bei $16 \mathrm{~mm}$, entsprechend $46,7 \% \%^{\circ}$ Eine bessere Ausbeute dürfte nach dem neuen Verfahren von H. Leuchs') die Anwendung eines groBen Überschusses von freiem Malonester liefern.

1) Carlo Marangolo, "Über die Einwirkung von Hydrazinhydrat auf $\mathrm{m}$-Xylylmalonsäurediäthylester". Inaug.-Diss. Heidelberg 1902. Druek von J. Hörning.

2) Ber. 23, 109 (1890).

s) Ber. 44, 1507 (1911). 